\title{
Prevention of progression of pulmonary hypertension by the Nur77 agonist 6-mercaptopurine: role of BMP signalling
}

\author{
Kondababu Kurakula ${ }^{1,7}$, Xiao-Qing Sun ${ }^{2,7}$, Chris Happé ${ }^{2}$ \\ Denielli da Silva Goncalves Bos ${ }^{2}$, Robert Szulcek ${ }^{2}$, Ingrid Schalij ${ }^{2}$, \\ Karien C. Wiesmeijer ${ }^{1}$, Kirsten Lodder ${ }^{1}$, Ly Tu ${ }^{3,4}$, Christophe Guignabert ${ }^{3,4}$, \\ Carlie J.M. de Vries ${ }^{5}$, Frances S. de Man ${ }^{2}$, Anton Vonk Noordegraaf ${ }^{2}$, \\ Peter ten Dijke ${ }^{6}$, Marie-José Goumans (10 ${ }^{1,8}$ and Harm Jan Bogaard ${ }^{2,8}$
}

Affiliations: ${ }^{1}$ Dept of Cell and Chemical Biology, Laboratory for Cardiovascular Cell Biology, Leiden University Medical Center, Leiden, The Netherlands. ${ }^{2}$ Pulmonary Medicine, Amsterdam Cardiovascular Sciences, Amsterdam UMC, Vrije Universiteit Amsterdam, Amsterdam, The Netherlands. ${ }^{3}$ INSERM UMR S 999, LabEx LERMIT, Hôpital Marie Lannelongue, Le Plessis-Robinson, France. 'Université Paris-Sud et Université Paris-Saclay, Le Kremlin-Bicêtre, France. ${ }^{5}$ Dept of Medical Biochemistry, Academic Medical Center, Amsterdam, The Netherlands. ${ }^{6}$ Dept of Cell and Chemical Biology, Oncode Institute, Leiden University Medical Center, Leiden, The Netherlands. ${ }^{7}$ These two authors contributed equally to this work. ${ }^{8}$ These two authors jointly supervised this work.

Correspondence: Harm Jan Bogaard, Pulmonary Medicine, Amsterdam Cardiovascular Sciences, Amsterdam UMC, Vrije Universiteit Amsterdam, De Boelelaan 1117, 1081 HV Amsterdam, The Netherlands.

E-mail: HJ.Bogaarddvumc.nl

@ERSpublications

Pharmacological activation of Nur77 with 6-mercaptopurine reduces the progression of pulmonary hypertension by enhancing BMP signalling http://bit.ly/2KINWCW

Cite this article as: Kurakula K, Sun X-Q, Happé C, et al. Prevention of progression of pulmonary hypertension by the Nur77 agonist 6-mercaptopurine: role of BMP signalling. Eur Respir J 2019; 54: 1802400 [https://doi.org/10.1183/13993003.02400-2018].

ABSTRACT Pulmonary arterial hypertension (PAH) is a progressive fatal disease characterised by abnormal remodelling of pulmonary vessels, leading to increased vascular resistance and right ventricle failure. This abnormal vascular remodelling is associated with endothelial cell dysfunction, increased proliferation of smooth muscle cells, inflammation and impaired bone morphogenetic protein (BMP) signalling. Orphan nuclear receptor Nur77 is a key regulator of proliferation and inflammation in vascular cells, but its role in impaired BMP signalling and vascular remodelling in PAH is unknown.

We hypothesised that activation of Nur77 by 6-mercaptopurine (6-MP) would improve PAH by inhibiting endothelial cell dysfunction and vascular remodelling.

Nur77 expression is decreased in cultured pulmonary microvascular endothelial cells (MVECs) and lungs of PAH patients. Nur77 significantly increased BMP signalling and strongly decreased proliferation and inflammation in MVECs. In addition, conditioned medium from PAH MVECs overexpressing Nur77 inhibited the growth of healthy smooth muscle cells. Pharmacological activation of Nur77 by 6-MP markedly restored MVEC function by normalising proliferation, inflammation and BMP signalling. Finally, 6-MP prevented and reversed abnormal vascular remodelling and right ventricle hypertrophy in the Sugen/hypoxia rat model of severe angioproliferative PAH.

Our data demonstrate that Nur77 is a critical modulator in PAH by inhibiting vascular remodelling and increasing BMP signalling, and activation of Nur77 could be a promising option for the treatment of PAH.

This article has supplementary material available from erj.ersjournals.com

Received: 18 Dec 2018 | Accepted after revision: 19 June 2019

Copyright @ERS 2019 


\section{Introduction}

Pulmonary arterial hypertension (PAH) is a progressive fatal disease caused by abnormal proliferation of pulmonary vascular cells, resulting in remodelling of small pulmonary arteries, increased pulmonary vascular resistance, right ventricular (RV) failure and ultimately death [1-4]. In hereditary PAH (HPAH), mutations in the gene encoding for bone morphogenetic protein receptor 2 (BMPR2) or in genes encoding for downstream SMAD transcriptional effectors lead to impaired BMP signalling [4-7]. Interestingly, impaired BMP signalling also occurs in nonhereditary forms of $\mathrm{PAH}[6,8]$. Despite advances in the treatment of $\mathrm{PAH}$, current therapies still fail to reverse the established abnormal remodelling present in the lungs. Therefore, new molecular targets for the development of remodelling focused therapeutics are urgently needed.

Pulmonary artery endothelial cells (PAECs), pulmonary artery smooth muscle cells (PASMCs), pericytes and fibroblasts all play crucial roles in the pathobiology of PAH [5, 9-12]. PAH PAECs are hyperproliferative and contribute to the decrease in luminal diameter of the pulmonary microvessels. Additionally, PAH PAECs secrete factors such as inflammatory cytokines and growth factors, which in turn induce proliferation and migration of PASMCs and fibroblasts, and contribute to the progression of PAH [13-17]. A loss of BMPR2 in the endothelium can initiate and sustain the development of PAH [18, 19], but the driving transcriptional mechanisms remain poorly understood. Here, we identify a central role for the orphan nuclear receptor Nur77 (nuclear receptor subfamily 4 group A member 1 (NR4A1)) in PAEC dysfunction, aberrant BMP signalling and vascular remodelling in PAH.

The transcription factor Nur77 plays a key role in a wide array of cellular processes such as proliferation, apoptosis and inflammation [20-25]. Nur77 is implicated in several cardiovascular diseases such as atherosclerosis, restenosis and cardiac hypertrophy $[20,22,26]$. In endothelial cells, Nur77 decreases endothelin-1 expression and attenuates pro-inflammatory responses via inhibition of the NF- $\kappa \mathrm{B}$ pathway $[27,28]$. Activation of Nur77 by cytosporone B (CsnB) was shown to ameliorate experimental pulmonary hypertension $(\mathrm{PH})$ in mice by decreasing PASMC proliferation [25, 29]. Interestingly, Nur77 modulates the transforming growth factor (TGF)- $\beta$ pathway in a cell- and context-dependent manner [30, 31]. 6-Mercaptopurine (6-MP), a well-established immunosuppressive drug to treat various autoimmune and chronic inflammatory diseases, is a nontraditional agonist of Nur77 [21, 22, 32]. 6-MP mediates a genotoxic stress response that in turn activates transcription of all three members of the NR4A nuclear hormone receptor family, i.e. Nur77, Nor1 and Nurr1 [32]. We and others have shown that 6-MP inhibits activation of immune cells, and also reduces the inflammatory response of endothelial cells and the proliferation of vascular smooth muscle cells in a Nur77-dependent manner [33-35]. Here, we show that Nur77 is a key player in PAH, involved in PAEC dysfunction, proliferation of PASMCs and impaired BMP signalling, and that pharmacological targeting of Nur77 by $6-\mathrm{MP}$ presents a novel therapeutic concept in PAH.

\section{Methods}

Detailed methods are provided in the supplementary material.

\section{Human lung samples and cell culture}

$\mathrm{PAH}$ lung tissues were collected with patient consent and approval from the local ethics committees at the Amsterdam UMC (Amsterdam, The Netherlands) and Comite dé Protection des Personnes (CPP) Ile-de-France VII (Paris, France). Control lung tissue was from patients undergoing a surgical procedure for cancer. Isolation and culturing of microvascular endothelial cells (MVECs) and PASMCs were performed as described previously [36, 37].

Plasmids, chemicals, quantitative real-time PCR, Western blot and luciferase assays

Please refer to the supplementary material for details.

Lentiviral transduction and proliferation assays

Lentiviral transduction of cells and cell proliferation (3-(4,5-dimethylthiazol-2-yl)-2,5-diphenyltetrazolium bromide (MTT)) assays were performed as described previously [21, 23].

\section{Sugen/hypoxia rat model of $\mathrm{PH}$}

Sugen (SU5416)/hypoxia (SuHx)-mediated PH in male Sprague-Dawley rats was induced as described previously $[38,39]$. In the prevention study, rats received 6-MP $\left(1 \mathrm{mg} \cdot \mathrm{kg}^{-1} \cdot \mathrm{day}^{-1}\right)$ in drinking water from day 0 to day 42. In the reversal study, after randomisation (treatment versus vehicle), animals were divided into two groups receiving 6-MP $\left(1\right.$ or $\left.7.5 \mathrm{mg} \cdot \mathrm{kg}^{-1} \cdot \mathrm{day}^{-1}\right)$ or vehicle (dimethyl sulfoxide) in drinking water from day 42 to day 70. A power calculation was performed to determine the appropriate sample size for both the prevention and reversal studies. Echocardiography, haemodynamic evaluation and RV 
hypertrophy assessment were blinded to the condition, followed an unbiased approach and were performed as described previously [38-40].

\section{Statistical analysis}

Statistical analyses were performed using Prism version 7 for Windows (GraphPad, La Jolla, CA, USA). The t-test was used for comparisons between two groups. Multiple comparisons were assessed by one-way ANOVA, followed by the Bonferroni post hoc test. p-values $<0.05$ were considered significant. All statistical tests used two-sided tests of significance. Data are presented as mean \pm SEM.

\section{Results}

\section{Nur77 expression is impaired in PAH MVECs}

Immunofluorescent staining showed that Nur77 is expressed in endothelial cells, smooth muscle cells, fibroblasts and epithelial cells in the lungs of control, idiopathic PAH (IPAH) and HPAH patients (figure 1a). Transcript levels of Nur77 were significantly decreased in lungs of both IPAH and HPAH compared with controls, as shown by quantitative real-time PCR (figure 1b). Consistent with this observation, mRNA levels of Nur77 were markedly decreased in cultured human pulmonary MVECs of both IPAH and HPAH compared with controls (figure 1c). Furthermore, protein levels of Nur77 were significantly decreased in MVECs of IPAH compared with controls (figure 1d). Taken together, these data suggest that Nur77 is involved in PAEC function in PAH.

\section{Nur77 modulates BMP/SMAD signalling in MVECs}

Confirming previous studies, levels of BMPR2 and Id1 were significantly decreased in IPAH MVECs compared with control cells (figure 1e). Ectopic expression of Nur77 modestly but significantly augmented BMPR2 mRNA levels in MVECs (figure 1f). Conversely, knockdown of Nur77 decreased BMPR2 protein levels in MVECs (figure 1g). To investigate the effect of Nur77 on canonical BMP signalling, we determined the phosphorylation of Smad1/5/8 (pSmad1/5/8) following ectopic expression of Nur77. Nur77 markedly increased pSmad1/5/8 under serum-, BMP9- and tumour necrosis factor (TNF)- $\alpha$-stimulated conditions (figure 1h). Ectopic expression of Nur77 augmented BMP9-induced BMP/ SMAD reporter (BRE-luciferase) activity [41] (figure 1i) and Id3 mRNA expression (figure 1j), while knockdown of Nur77 decreased BMP9-induced BRE-luciferase activity in MVECs (figure 1k). Nur77 also augmented the expression level of Id1 in PAH MVECs (figure 11). Taken together, our data demonstrate that Nur77 increases BMP/SMAD signalling in MVECs.

\section{Nur77 reduces inflammation of MVECs by inhibiting NF- $\kappa B$ activity}

As expected, PAH MVECs have higher mRNA levels of the pro-inflammatory cytokines interleukin (IL)-6 and RANTES after TNF- $\alpha$ stimulation when compared with control cells (figure 2a). Knockdown of Nur77 markedly increased TNF- $\alpha$-induced expression of TNF- $\alpha$, IL-1 $\beta$ and IL- 6 at the mRNA level (figure 2b), while ectopic expression of Nur77 decreased the protein levels of monocyte chemoattractant protein (MCP)-1 and IL-6 in MVECs (figure 2c and d). Furthermore, knockdown of Nur77 significantly increased (figure 2e), while ectopic expression of Nur77 strongly decreased, the transcriptional activity of the NF- $\mathrm{\kappa B}$ promoter (figure 2f), suggesting that in pulmonary artery MVECs Nur77 decreases the expression of pro-inflammatory cytokines through inhibition of the NF- $\kappa \mathrm{B}$ pathway.

\section{Nur77 attenuates proliferation of MVECs through inhibiting cyclin D1}

Cultured MVECs from both control and PAH patients display a cobblestone morphology (figure $2 \mathrm{~g}$ ). However, PAH MVECs grow faster as demonstrated by MTT assay (figure 2h). Since Nur77 is reported to decrease cell proliferation by inhibiting cyclin D1 [25], we explored whether loss of Nur77 could explain the hyperproliferation of PAH MVECs. Overexpression of Nur77 reduced proliferation of both PAH and control MVECs (figure 2h), while knockdown of Nur77 stimulated their growth. Furthermore, we found that overexpression of Nur77 decreased (figure 2j), while knockdown of Nur77 significantly increased, cyclin D1 promoter activity (figure 2k). Taken together, these data suggest that Nur77 is involved in PAH MVEC proliferation via modulation of cyclin D1.

Since the interplay between endothelial cells and smooth muscle cells is crucial in the pathogenesis of $\mathrm{PAH}$, we next investigated the role of Nur77 in endothelial cell-smooth muscle cell interaction. Addition of conditioned medium from TNF- $\alpha$-pre-treated PAH PAECs transduced with Nur77 inhibited the growth of healthy PASMCs (figure 21), indicating that Nur77 plays an important role not only in PAECs but also in the cross-talk between endothelial cells and smooth muscle cells. 


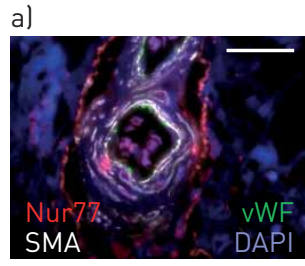

Control

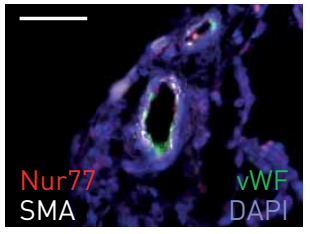

IPAH

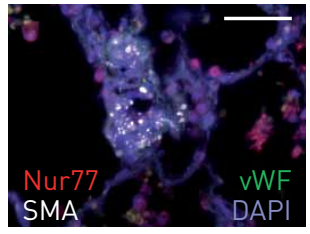

HPAH d)

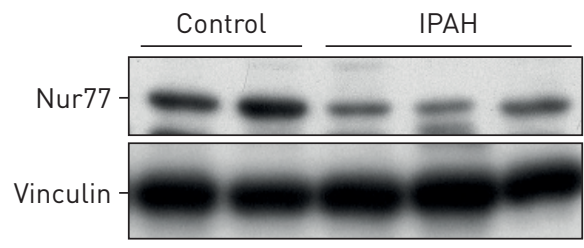

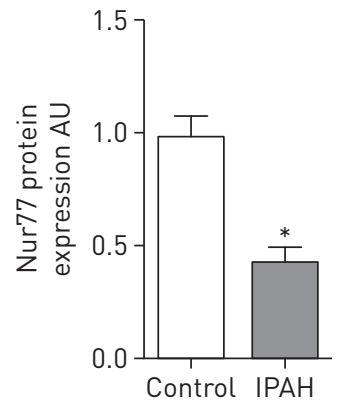
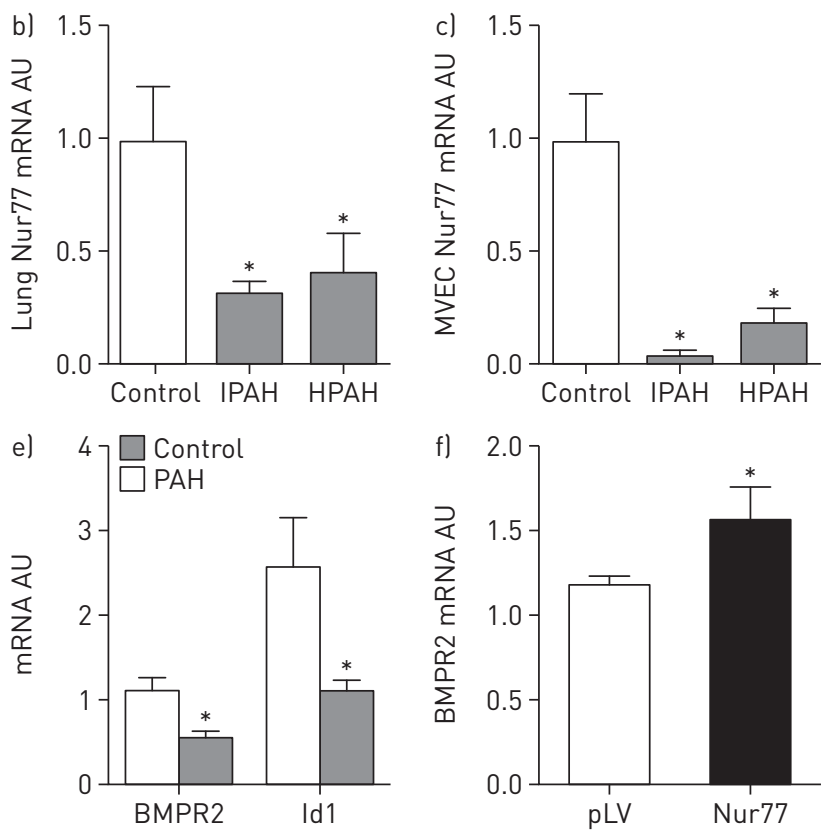

g)
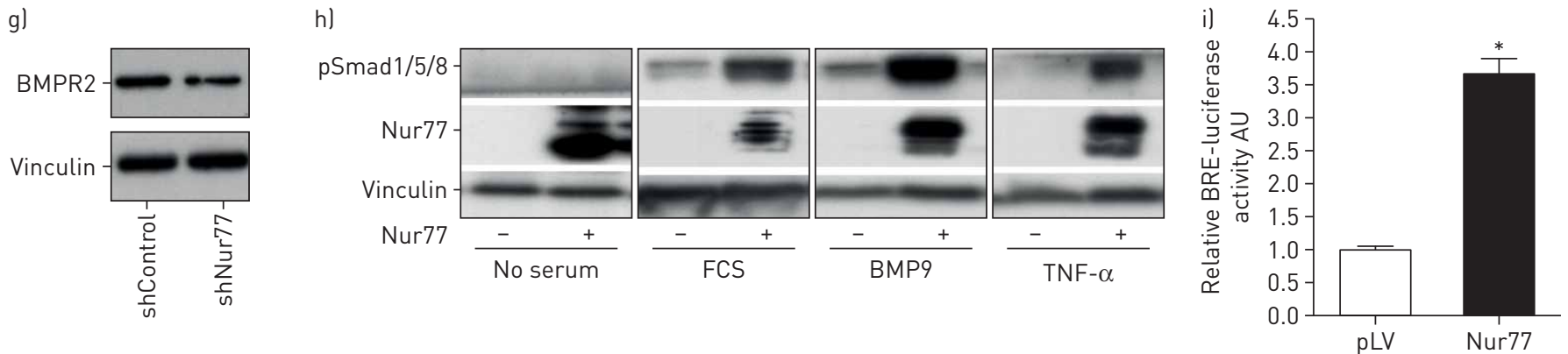
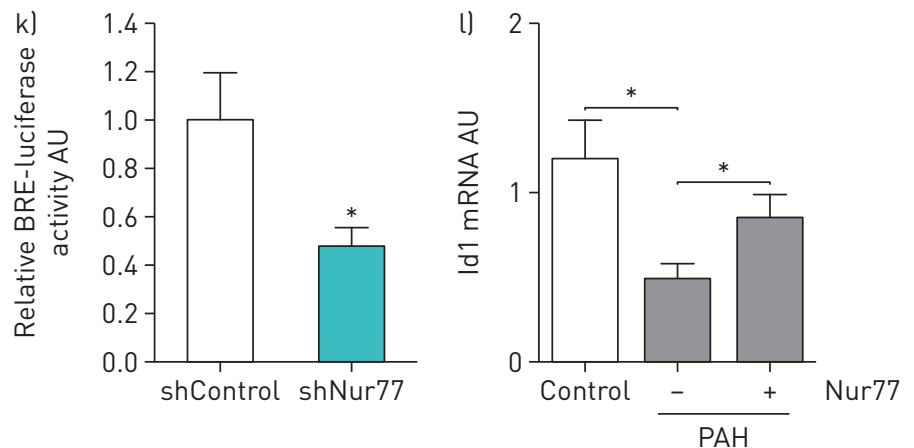

FIGURE 1 Nur77 expression is reduced in pulmonary arterial hypertension (PAH) and Nur77 enhances bone morphogenetic protein (BMP) signalling in microvascular endothelial cells (MVECs). vWF: von Willebrand factor; DAPI: 4',6-diamidino-2-phenylindole; SMA: $\alpha$-smooth muscle actin; IPAH: idiopathic PAH; HPAH: hereditary PAH; AU: arbitrary units; BMPR2: BMP receptor 2; pLV: control lentivirus; sh: short hairpin; pSmad1/5/8: phosphorylated Smad1/5/8; TNF: tumour necrosis factor; qRT: quantitative real-time. a) Representative immunofluorescence photomicrographs of Nur77 (red), vWF (green) and SMA (white) in human pulmonary arteries from control, IPAH and HPAH lungs (n=4). DAPI (blue): nuclei. Scale bar: $50 \mu \mathrm{m}$. b, c) qRT-PCR was performed to assess mRNA expression of Nur77 in b) control, IPAH and HPAH lungs ( $\mathrm{n}=6$ per group) and in c) MVECs from control, IPAH and HPAH patients ( $n=4$ per group). d) Representative Western blots with relative densitometric analyses showing Nur77 in total lung from control and IPAH patients ( $n=3$ ). e) qRT-PCR was performed to assess mRNA expression of BMPR2 and Id1 in MVECs from control and PAH patients ( $n=6$ per group). f) qRT-PCR was performed to assess mRNA expression of BMPR2 in MVECs following overexpression of Nur77 (n=3). g) Representative Western blots showing BMPR2 in MVECs following knockdown of Nur77 by shNur77 lentivirus (n=3). h) Representative Western blots showing pSmad1/5/8 following overexpression of Nur77 and stimulation with vehicle (no serum), serum (10\% FCS), BMP9 (1 $\mathrm{ng} \cdot \mathrm{mL}^{-1}$ ) and TNF- $\alpha$ ( $50 \mathrm{ng} \cdot \mathrm{mL}^{-1}$ ) in MVECs (n=3). i) BRE-luciferase activity in MVECs was measured following overexpression of Nur77 and stimulation with BMP9 (1 $\left.\mathrm{ng} \cdot \mathrm{mL}^{-1}\right)$ for $16 \mathrm{~h}(\mathrm{n}=3)$. j) qRT-PCR was performed to assess mRNA expression of Id3 in MVECs following ectopic expression of Nur77 (n=3). k) BRE-luciferase activity in MVECs was measured following knockdown of Nur77 and stimulation with BMP9 (1 $\mathrm{ng} \cdot \mathrm{mL}^{-1}$ ) for $16 \mathrm{~h}(\mathrm{n}=3)$. l) qRT-PCR was performed to assess mRNA expression of Id1 in control and PAH MVECs following ectopic expression of Nur77 ( $n=3$ ). *: $p<0.05$. The t-test was used for comparisons between two groups. Multiple comparisons were assessed by one-way ANOVA followed by Bonferroni's post hoc test. Data are presented as mean \pm SEM. 

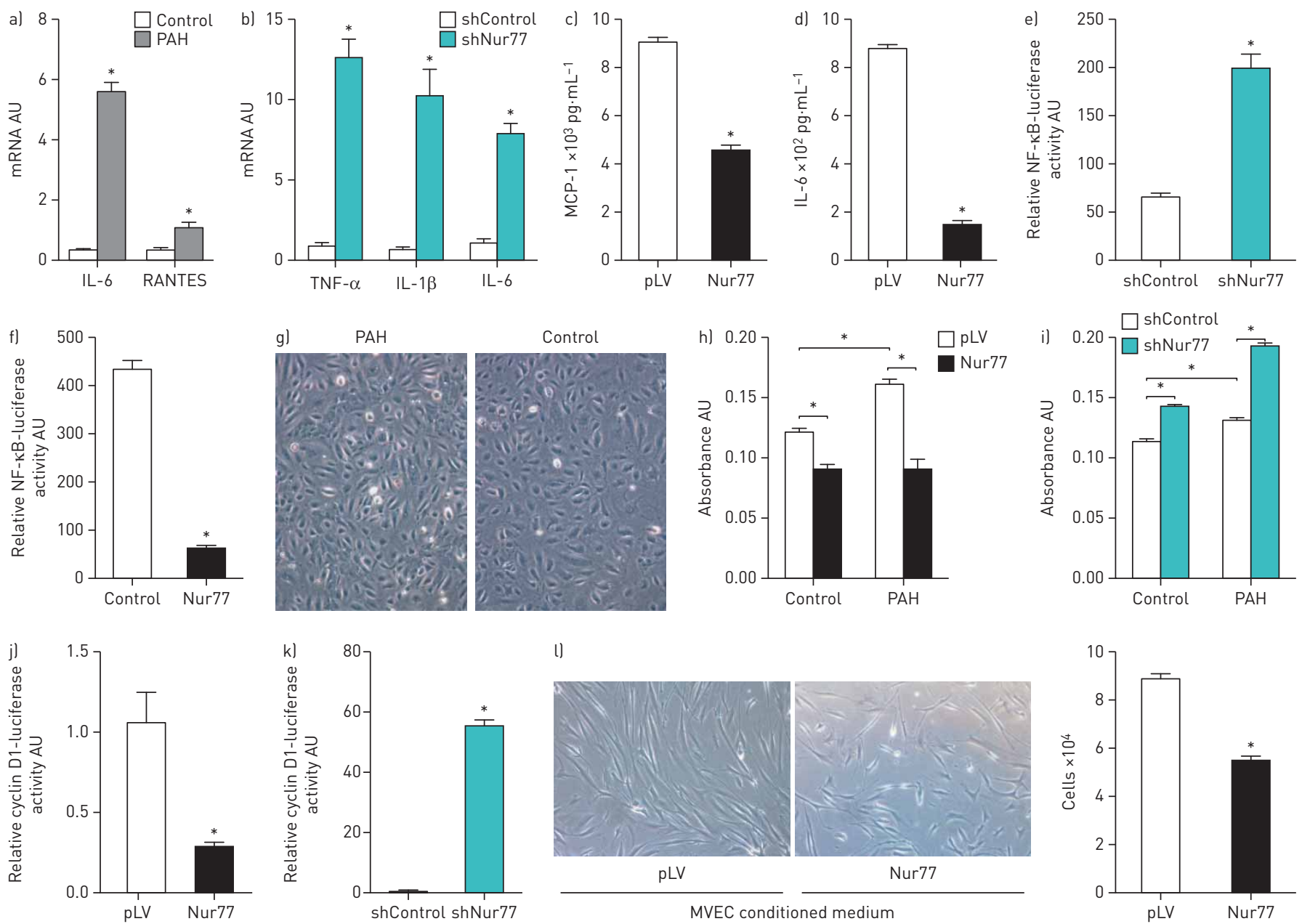

FIGURE 2 Nur77 inhibits inflammation and proliferation of microvascular endothelial cells (MVECs). AU: arbitrary units; PAH: pulmonary arterial hypertension; IL: interleukin; sh: short hairpin; TNF: tumour necrosis factor; MCP: monocyte chemoattractant protein; pLV: control lentivirus: qRT: quantitative real-time; MTT: 3-(4,5-dimethylthiazol-2-yl)-2,5-diphenyltetrazolium bromide. a) qRT-PCR was performed to assess mRNA expression of IL- 6 and RANTES in TNF- $\alpha$-stimulated MVECs $(6 \mathrm{~h})$ from control and idiopathic PAH patients (n=3). b) qRT-PCR was performed to assess mRNA expression of TNF- $\alpha$, IL-1 $\beta$ and IL- 6 in TNF- $\alpha$-stimulated MVECs ( 6 h) ( $n=3$ ). c, d) ELISAs for c) MCP- 1 and d) IL- 6 were performed using supernatants from MVECs following ectopic expression of Nur77 and stimulation with TNF- $\alpha$ for $6 \mathrm{~h}(\mathrm{n}=4$ ). e, f) TNF- $\alpha$-induced NF-KB-luciferase activity in HEK293T cells was measured following e) knockdown and f) ectopic expression of Nur77 ( $\mathrm{n=3}$ ). g) Morphological appearance of control and PAH MVECs. h, i) MTT assays were performed to assess proliferation of control and PAH MVECs following h) knockdown and i) ectopic expression of Nur77 (n=3). j, k) Cyclin D1 promoter-luciferase activity in HEK293T cells was measured following j) ectopic expression and kJ knockdown of Nur77. The data shown are representative of three experiments. I) Assessment of serum-starved pulmonary artery smooth muscle cell proliferation following incubation with conditioned medium from PAH MVECs without and with ectopically expressed Nur77 and stimulated with TNF- $\alpha(n=3) . *$ : $p<0.05$. The t-test was used for comparisons between two groups. Multiple comparisons

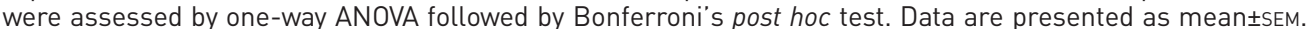

Nur77 is required for 6-MP-induced activation of BMP signalling

To increase Nur77 activity we stimulated PAH MVECs with two known agonists of Nur77, i.e. CsnB and 6-MP [21, 42], and found that both agonists increased the protein levels (figure 3a) and mRNA expression of Nur77 (figure 3b) in PAH MVECs. Since the transcriptional activity of Nur77 determines its function, we determined the transcriptional activity of Nur77 using a Nur77-specific luciferase reporter (NurRE-luciferase) [23] in MVECs and observed that both agonists increased the activity of the Nur77 reporter (figure 3c). Interestingly, $\mathrm{CsnB}$ and 6-MP also significantly increased BRE-luciferase activity (figure 3d). To determine whether the observed increase in BRE-luciferase activity required Nur77, we knocked down Nur77 and found that both agonists were no longer able to activate the BMP reporter (figure 3d). This confirmed our previous observation that Nur77 activates the BMP/SMAD signalling pathway. In line with the increased BRE-luciferase activity, both agonists also increased the mRNA levels of the downstream target Id1 (figure 3e). Moreover, treatment of PAH MVECs with both agonists decreased proliferation (figure 3f) and attenuated cyclin D1 promoter-luciferase activity (figure $3 \mathrm{~g}$ ). We also found that both $\mathrm{CsnB}$ and 6-MP require Nur77 to reduce pro-inflammatory cytokine IL-6 expression 
a)

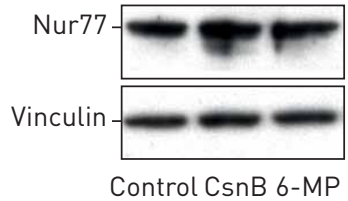

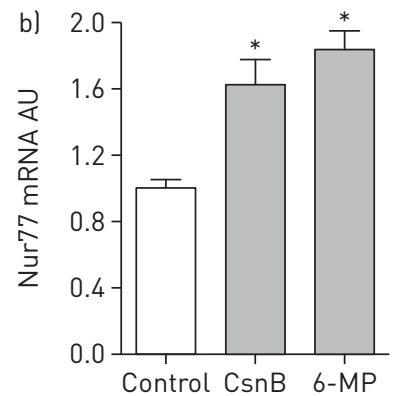
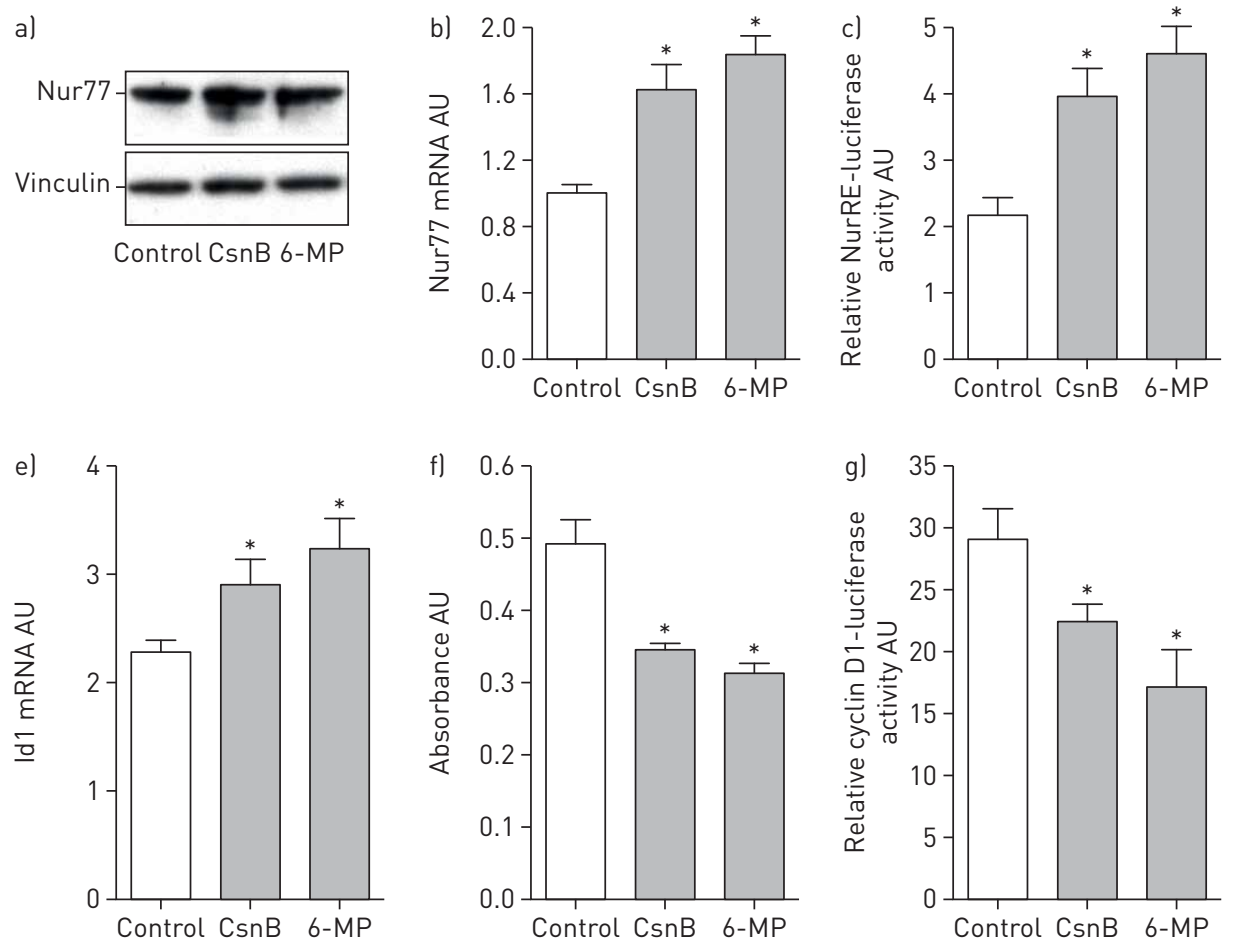

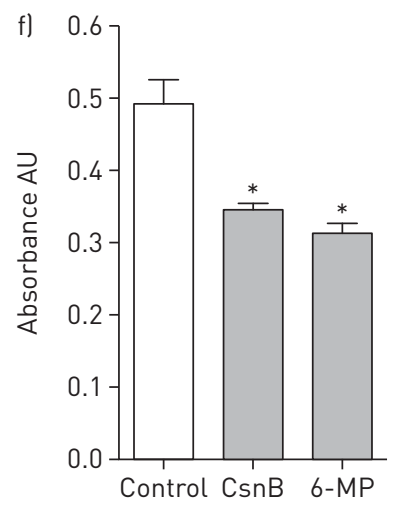

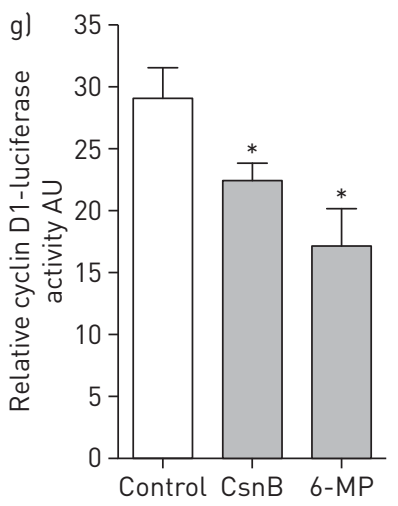

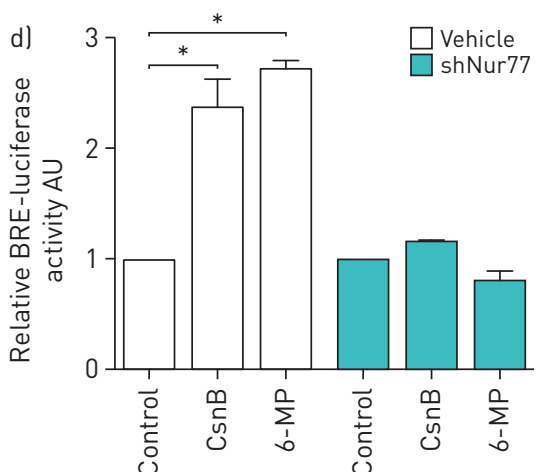

FIGURE 3 Upregulated Nur77 is involved in the 6-mercaptopurine (6-MP)-mediated effect on bone morphogenetic protein (BMP) signalling in microvascular endothelial cells (MVECs). CsnB: cytosporone B; AU: arbitrary units; qRT: quantitative real-time; MTT: 3-(4,5-dimethylthiazol-2-yl)-2,5-diphenyltetrazolium bromide; PAH: pulmonary arterial hypertension. a) Western blot (n=3) and b) qRT-PCR ( $\mathrm{n}=3$ ) analysis was performed to measure the expression of Nur77 in MVECs that were pre-treated with CsnB (5 $\mu \mathrm{M})$ or $6-\mathrm{MP}$ (10 $\mu \mathrm{M})$ for $16 \mathrm{~h}$. c) The activity of Nur77 was monitored in MVECs expressing the NurRE-luciferase reporter following treatment with $\mathrm{CsnB}(5 \mu \mathrm{M})$ or $6-\mathrm{MP}(10 \mu \mathrm{M})$. The data shown are representative of three experiments. d) Overall BRE-luciferase activity was measured in MVECs transduced with control or shNur77 lentivirus for $48 \mathrm{~h}$ followed by treatment with $\operatorname{CsnB}(5 \mu \mathrm{M})$ or $6-\mathrm{MP}(10 \mu \mathrm{M})$ in the presence of $\mathrm{BMP}$ (1 $\left.\mathrm{ng} \cdot \mathrm{mL}^{-1}\right)$ for $16 \mathrm{~h}(\mathrm{n}=3)$. e) qRT-PCR was performed to assess mRNA expression of Id 1 following treatment with CsnB (5 $\mu \mathrm{M})$ or $6-\mathrm{MP}(10 \mu \mathrm{M})$ for $16 \mathrm{~h}$ (n=3). f) MTT assays were performed to assess PAH MVEC proliferation following treatment with CsnB $(5 \mu \mathrm{M})$ or $6-\mathrm{MP}(10 \mu \mathrm{M})$ for $24 \mathrm{~h}$ ( $\mathrm{n}=3)$. g) Cyclin $\mathrm{D} 1$ promoter-luciferase activity in PAH MVECs was measured following treatment with CsnB $(5 \mu \mathrm{M})$ or $6-\mathrm{MP}(10 \mu \mathrm{M})$ for $16 \mathrm{~h}$ (n=3). *: p<0.05. Multiple comparisons were assessed by one-way ANOVA followed by Bonferroni's post hoc test. Data are presented as mean \pm SEM.

(data not shown). Taken together, we show that both $\mathrm{CsnB}$ and 6-MP require Nur77, at least partly, to exhibit their function in restoring the behaviour of PAH MVECs. Although both agonists of Nur77 displayed similar potential in vitro, we assessed whether 6-MP could prevent development of $\mathrm{PH}$ in the SuHx rat model for angioproliferative PH [43], because 6-MP has 1) been used in the clinic for decades $[21,44], 2)$ decreased inflammation in cultured MVECs (supplementary figure S1a and b), 3) increased the expression levels of Id3 and BMPR2 in PAH MVECS (supplementary figure S1c and d), and 4) decreased proliferation of healthy human PASMCs (supplementary figure S1e).

\section{6-MP treatment prevents the development of $\mathrm{PH}$}

When treating the animals with $1 \mathrm{mg} \cdot \mathrm{kg}^{-1} 6-\mathrm{MP}$ per day orally from the start of the experiment till day 42 we found that 6-MP was able to maintain RV function by significantly decreasing RV systolic pressure (RVSP), arterial elastance $\left(E_{\mathrm{a}}\right)$ and RV end-diastolic elastance (stiffness, $E_{\text {ed }}$ ), while increasing RV-arterial coupling (end-systolic elastance $\left(E_{\mathrm{es}}\right) / E_{\mathrm{a}}$ ) (figure $4 \mathrm{a}$ ). Moreover, 6-MP treatment significantly increased stroke volume (SV) and RV systolic function (tricuspid annular plane systolic excursion (TAPSE)), while significantly decreasing RV wall thickness (RVWT) and RV end-diastolic diameter (RVEDD) (figure 4b). Consistent with our in vitro findings, 6-MP treatment also decreased serum levels of the inflammatory cytokines IL-6 and MCP-1 (figure 4c). However, no significant effect of 6-MP on the levels of phospho-IкB kinase- $\alpha / \beta$ was observed (supplementary figure S2a). Furthermore, 6-MP significantly reduced the number of occlusive lesions and fully muscularised arteries, and reduced the thickness of both the intimal and medial layers (figure $4 \mathrm{~d}$ and data not shown). The number of proliferating cells was decreased in rats treated with 6-MP compared with vehicle, as determined by immunofluorescent staining for Ki-67 (figure 4e). Furthermore, 6-MP treatment attenuated the recruitment of $\mathrm{CD}^{+} 8^{+}$macrophages to the lungs (figure 4f), while no significant effect of 6-MP was observed on the number of B-cells and T-cells (supplementary figure S2b and c). We found no evidence of 6-MP changing the composition of 

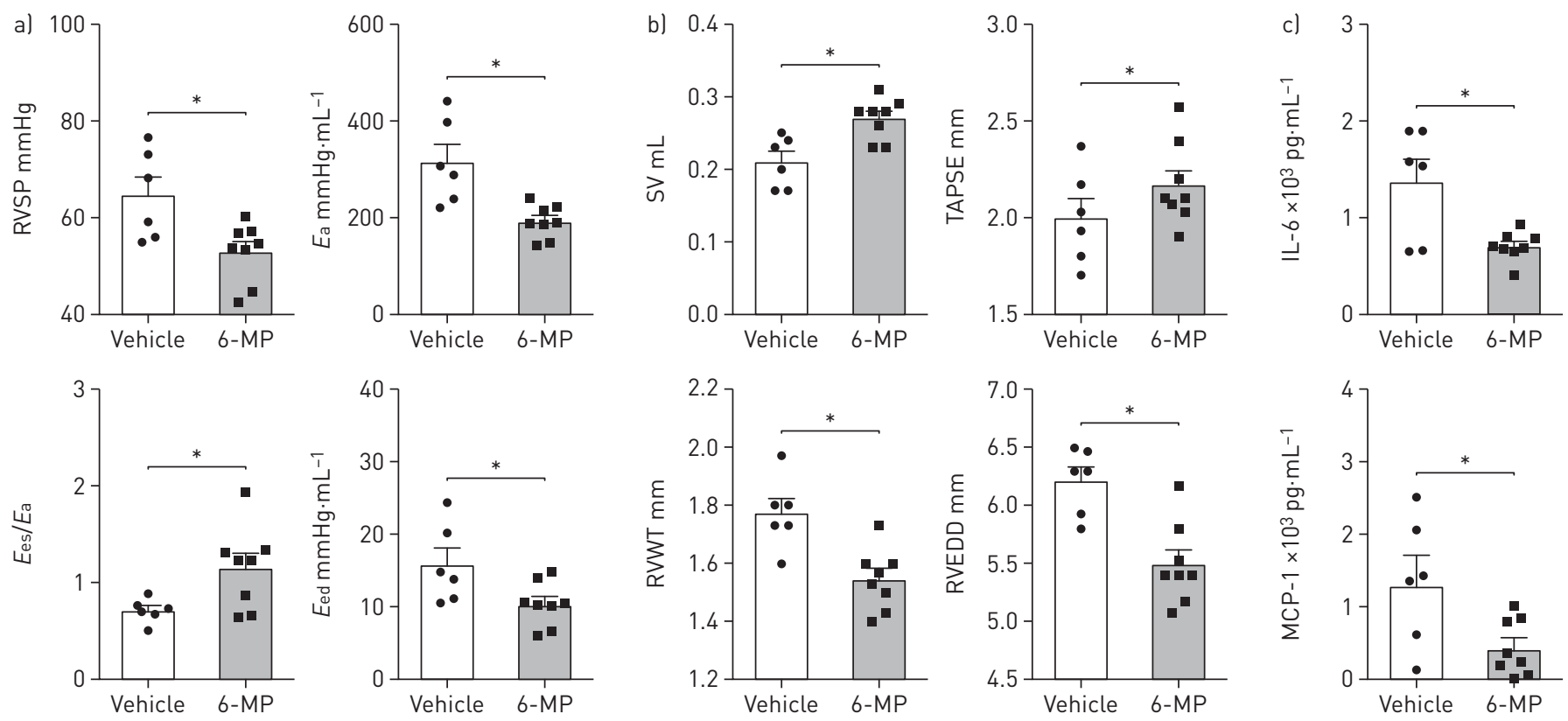

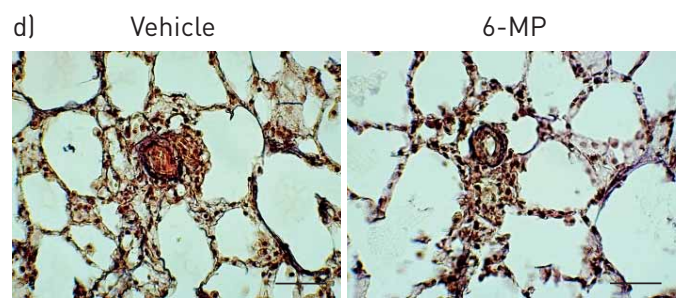

e)

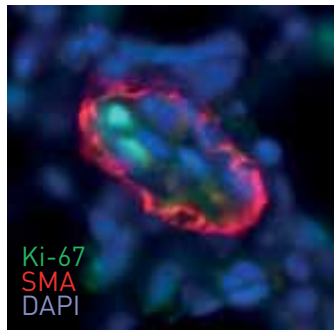

f)
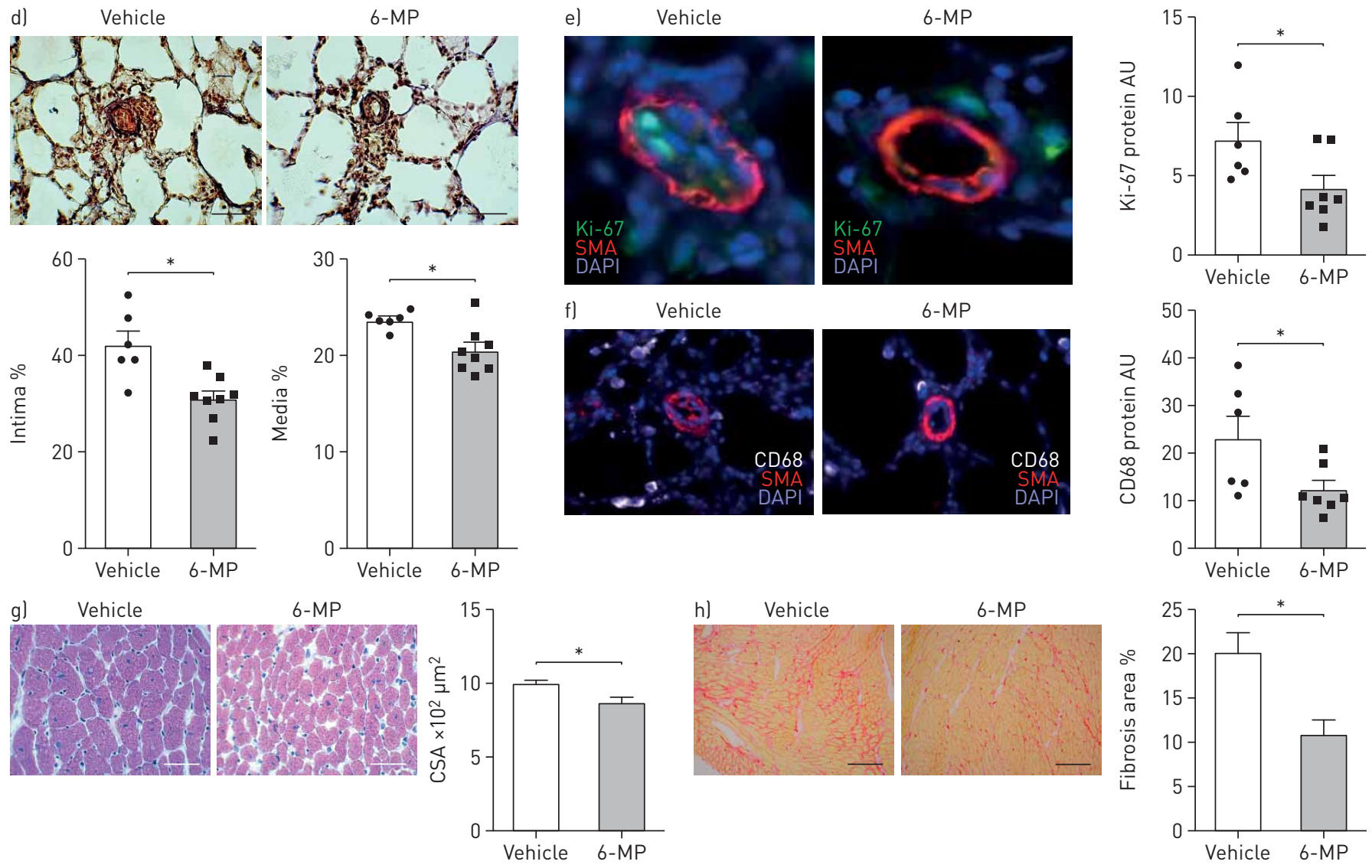

h) Vehicle

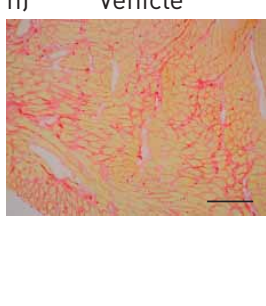

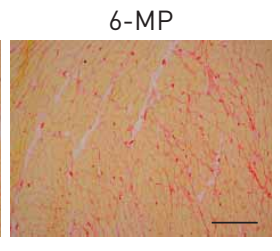

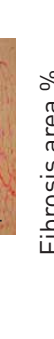

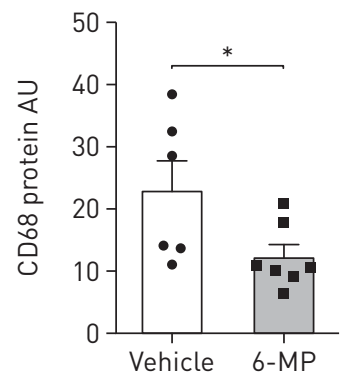

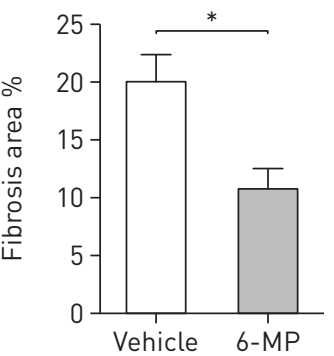

FIGURE 4 6-Mercaptopurine (6-MP) prevents the development of pulmonary hypertension (PH) and improves cardiac function in Sugen/hypoxia (SuHx)-induced PH in rats. RVSP: right ventricular systolic pressure; $E_{\mathrm{a}}$ : arterial elastance; $E_{\mathrm{es}}$ : end-systolic elastance; $E_{\mathrm{ed}}$ : end-diastolic elastance; SV: stroke volume; TAPSE: tricuspid annular plane systolic excursion; RVWT: right ventricular wall thickness; RVEDD: right ventricular end-diastolic diameter; IL: interleukin; MCP: monocyte chemoattractant protein; SMA: $\alpha$-smooth muscle actin; DAPI: 4',6-diamidino-2-phenylindole; AU: arbitrary units; CSA: cross-sectional area. a) SuHx rats were treated with 6-MP in drinking water from the start till the end of the experiment. Echocardiography, followed by physiological measurements, was carried out at the day of termination (day 42). RVSP (top left), $E_{\mathrm{a}}$ (top right), $E_{\mathrm{es}} / E_{\mathrm{a}}$ (bottom left) and $E_{\mathrm{ed}}$ (bottom right) are shown for the indicated groups of rats ( $\mathrm{n}=6$ rats for vehicle and $\mathrm{n}=8$ rats for 6-MP per group). b) SV (top left), TAPSE (top right), RVWT (bottom left) and RVEDD (bottom right) are shown for the indicated groups ( $n=6-8$ lungs per group). c) ELISAs for IL-6 (top) and MCP-1 (bottom) were performed in serum from the indicated groups ( $n=6-8$ lungs per group). d) The neointima to media ratio of pulmonary arteries $(<90 \mu \mathrm{m}$ in diameter) from lung sections of SuHx-treated rats was determined by 
morphometric analysis of pulmonary vessels following Elastica van Giesson staining ( $n=6-8$ lungs per group). Scale bar: $20 \mu m$. e, $f$ ) Immunofluorescent staining for e) Ki-67 (green) and f) CD68 (white) was performed in small pulmonary arteries from vehicle- and 6-MP-treated rats. SMA (red) and nuclei (DAPI; blue) were co-stained with Ki-67 or CD68. Quantification of fluorescence is shown in the bar graph next to the images (images representative of $n=6-8$ lungs per group). g) Mean cardiomyocyte CSA was assessed in the right ventricle from vehicle- and 6-MP-treated rats following haematoxylin and eosin staining. Scale bar: $20 \mu \mathrm{m}$. Quantification of CSA is shown in the bar graph next to the images (n=6-8 lungs per group). h) Picrosirius red staining was performed to assess fibrosis in the right ventricle from vehicle- and 6-MP treated rats. Scale bar: $80 \mu \mathrm{m}$. Quantification of fibrosis is shown in the bar graph next to the images ( $n=6-8$ lungs per group). *: $p<0.05$. The $t$-test was used for comparisons between two groups. Data are presented as mean \pm SEM.

blood cells or systemic blood pressure (supplementary figure S2d and data not shown). Finally, but importantly, RV hypertrophy and RV fibrosis were significantly decreased in 6-MP-treated PH rats (figure $4 \mathrm{~g}$ and $\mathrm{h}$ ). Taken together, 6-MP treatment from the start of the experiment prevents the development of $\mathrm{PH}$ in the SuHx rat model.

\section{6-MP treatment reverses the development of $\mathrm{PH}$, restores BMP signalling and increases Nur77 in vivo}

Before starting 6-MP treatment during disease progression, we first determined the expression levels of Nur77 in the SuHx model and observed that the expression of Nur77 is increased at initiation of the disease, while its expression decreases when disease progresses from week 6 onwards (supplementary figure S3). However, in the monocrotaline rats Nur77 levels remained elevated until the usual day of end-experiments: day 21 (data not shown) [25]. Therefore, to assess the therapeutic potential of 6-MP in $\mathrm{PH}$, we treated SuHx rats with two doses of $6-\mathrm{MP}\left(1\right.$ and $\left.7.5 \mathrm{mg} \cdot \mathrm{kg}^{-1} \cdot \mathrm{day}^{-1}\right)$ from day 28 after the induction of PH. The therapeutic dose of $6-\mathrm{MP}\left(7.5 \mathrm{mg} \cdot \mathrm{kg}^{-1} \cdot \mathrm{day}^{-1}\right)$ significantly decreased RVSP, $E_{\mathrm{a}}$ and $E_{\text {ed }}$, but not $E_{\text {es }} / E_{\mathrm{a}}$ (figure $5 \mathrm{a}$ ), while the subclinical dose of 6-MP used in the prevention trial $\left(1 \mathrm{mg} \cdot \mathrm{kg}^{-1} \cdot \mathrm{day}^{-1}\right)$ only significantly decreased $E_{\text {ed }}$, but had no effect on the other parameters (figure $5 \mathrm{a}$ ). The therapeutic dose of 6-MP markedly improved RV function (increase in SV), reduced RV hypertrophy (decrease in RVWT, RVEDD and Fulton index) and suppressed remodelling of the lung vasculature (figure $5 \mathrm{~b}-\mathrm{d}$ ). However, no significant effect of the therapeutic dose of 6-MP was observed on TAPSE, but the subclinical dose of 6-MP exhibited an increase in SV and a modest decrease in RVWT and RVEDD (figure $5 \mathrm{~b}$ ). Similar to the prevention study, the therapeutic dose of 6-MP decreased cell proliferation (number of Ki-67-positive cells) (figure 5e), RV hypertrophy (cross-sectional area) (figure 5f) and RV fibrosis (Picrosirius red staining) (figure 5g). In addition, we found that 6-MP significantly increased RV capillary density when compared with vehicle-treated animals (supplementary figure S4). Importantly, we found no evidence of 6-MP affecting blood cell composition or systemic blood pressure (supplementary figure S5a-c and data not shown), but it did improve the survival rate of the SuHx rats (supplementary figure S5d).

We next examined the effect of 6-MP treatment on BMP signalling in the lungs of SuHx rats. In the prevention study, we found that 6-MP increased the expression levels of BMPR2, pSmad1/5/8 and Id 3 as demonstrated by immunofluorescent analysis (figure 6a-c). Furthermore, 6-MP treatment significantly increased the expression levels of Nur77 in the vessel wall (figure 6d). However, no effect of 6-MP on Nur77 in total lung tissue was found as shown by Western blot analysis (supplementary figure S6). Consistent with the aforementioned findings, the therapeutic dose of 6-MP was able to increase the protein levels of BMPR2, pSmad1/5/8, Id1 and $\mathrm{Id} 3$ in the reversal study (figure $6 \mathrm{e}$ and $\mathrm{f}$, and supplementary figure S7a). However, no significant effect of 6-MP on phosphorylated extracellular signal-regulated kinase-1/2 or apoptosis was observed (supplementary figure S7b and c). Immunofluorescent analysis showed that 6-MP increased the expression levels of Nur77 in the vessel wall (figure $6 \mathrm{~g}$ ), while a modest increase was observed in total lung lysates (figure $6 \mathrm{e}$ and $\mathrm{f}$ ). Taken together, 6-MP augmented BMP signalling in vivo, at least partly, via Nur77. We summarise our findings in figure $6 \mathrm{~h}$.

\section{Discussion}

The present study demonstrates that downregulation of Nur77 in the pulmonary vasculature is involved in the pathogenesis of $\mathrm{PAH}$, and that activation of Nur77 by 6-MP could be a novel therapeutic option to reverse the abnormal vascular remodelling and RV hypertrophy in PAH. This concept (figure 6h) is based on our findings that 1) Nur77 expression is downregulated in both pulmonary MVECs and lungs of IPAH and HPAH patients, 2) absence of Nur77 leads to endothelial cell dysfunction and PASMC proliferation, 3) Nur77 suppresses inflammation through inhibition of the NF- $\mathrm{KB}$ pathway, and 4) pharmacological activation or gain of function of Nur77 inhibits MVEC dysfunction and potently augments BMP/SMAD signalling in MVECs and lungs in vitro and in vivo. Finally, 5) we demonstrated that pharmacological activation of Nur77 attenuates abnormal vascular remodelling and improves RV function in vivo. 

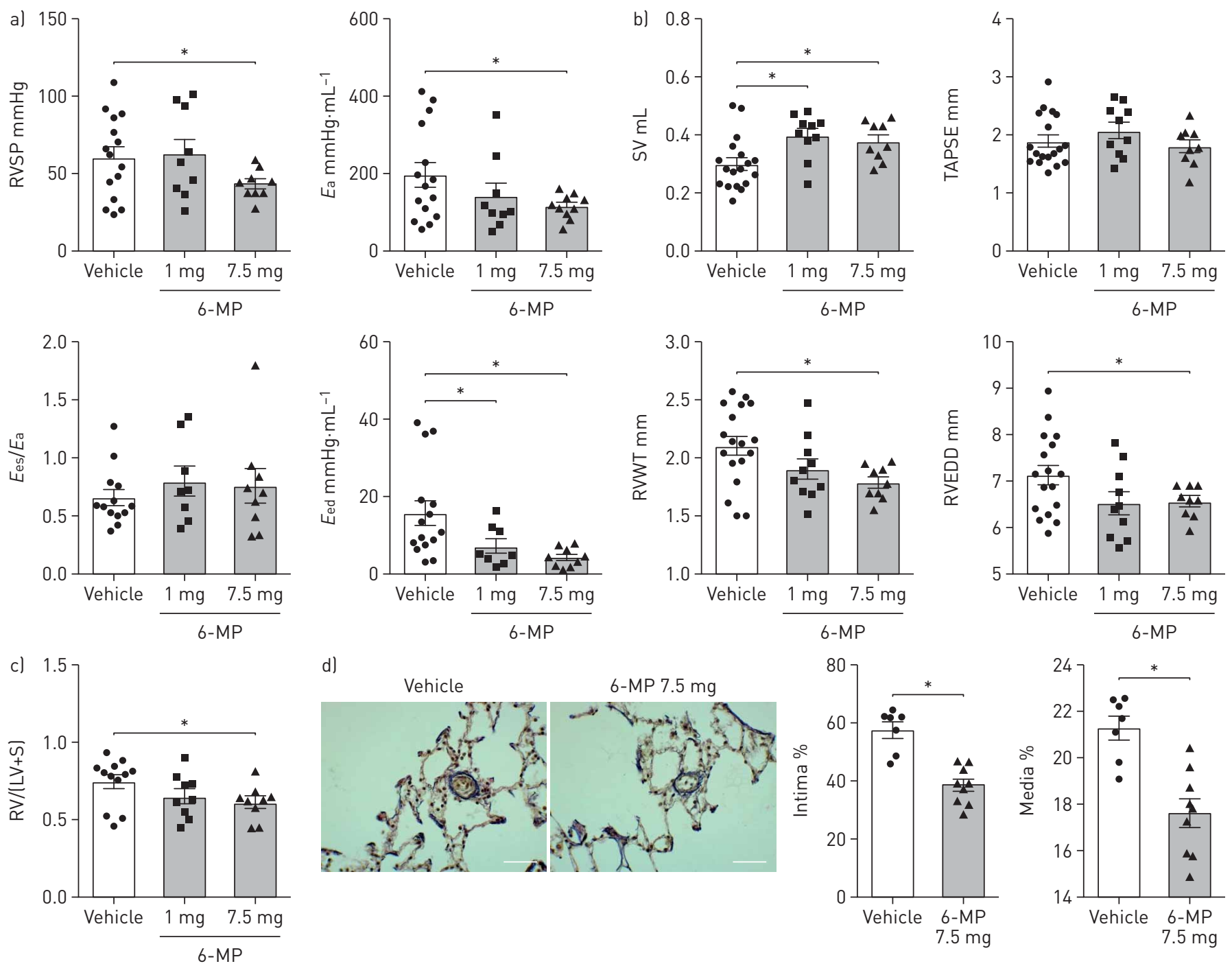

d)
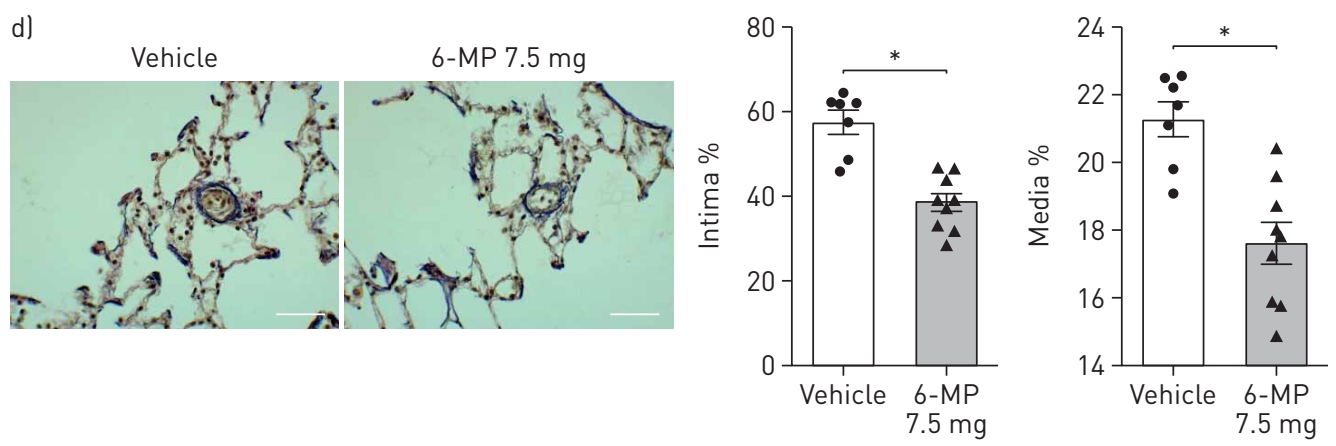

e) Vehicle

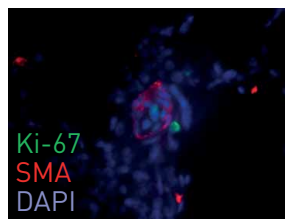

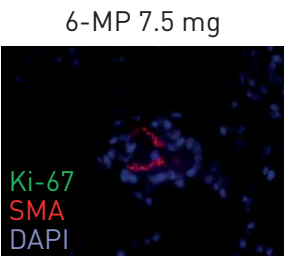
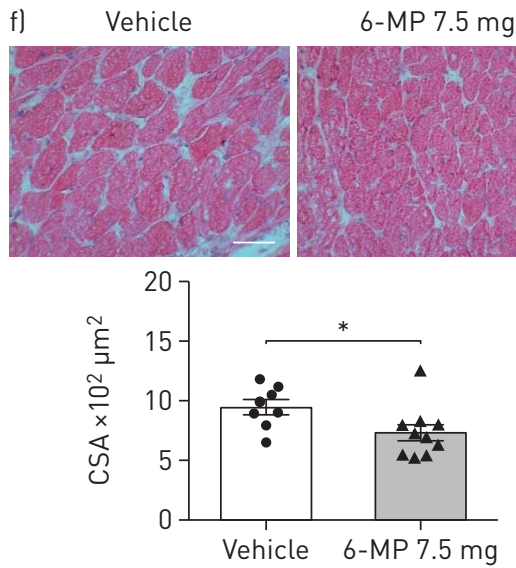

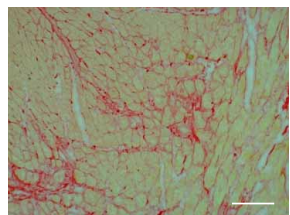

6-MP $7.5 \mathrm{mg}$
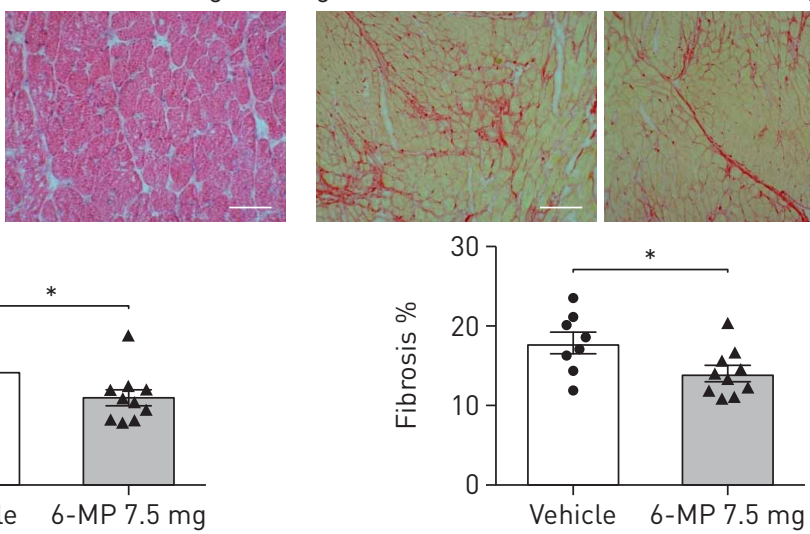

FIGURE 5 6-Mercaptopurine (6-MP) reverses pulmonary vascular remodelling and pulmonary hypertension (PH) in Sugen/hypoxia (SuHx)-induced $\mathrm{PH}$ in rats. RVSP: right ventricular systolic pressure; $E_{\mathrm{a}}$ : arterial elastance; $E_{\mathrm{es}}$ : end-systolic elastance; $E_{\mathrm{ed}}$ : end-diastolic elastance; SV: stroke volume; TAPSE: tricuspid annular plane systolic excursion; RVWT: right ventricular wall thickness; RVEDD: right ventricular end-diastolic diameter; RV: right ventricle; LV: left ventricle; S: septum; SMA: $\alpha$-smooth muscle actin; DAPI: 4',6-diamidino-2-phenylindole; CSA: cross-sectional area. a) Two concentrations $\left(1\right.$ and $\left.7.5 \mathrm{mg} \cdot \mathrm{kg}^{-1} \cdot \mathrm{day}^{-1}\right)$ of $6-\mathrm{MP}$ were administered in the drinking water from day 42 to day 70 to SuHx rats after randomisation. Echocardiography, followed by physiological measurements, was carried out at the day of termination (day 70 ). RVSP (top left), $E_{\mathrm{a}}$ (top right), $E_{\mathrm{es}} / E_{\mathrm{a}}$ (bottom left) and $E_{\mathrm{ed}}$ (bottom right) are shown for the indicated groups of rats ( $\mathrm{n}=14 \mathrm{rats}$ for vehicle or $\mathrm{n}=9$ for 6-MP $1 \mathrm{mg} \cdot \mathrm{kg}^{-1}$. day ${ }^{-1}$ or $\mathrm{n}=9$ for $6-\mathrm{MP} 7.5 \mathrm{mg} \cdot \mathrm{kg}^{-1} \cdot \mathrm{day}^{-1}$ per group). b) SV (top left), TAPSE (top right), RVWT (bottom left) and RVEDD (bottom right) are shown for the indicated groups ( $n=9-14$ rats per group). c) Right ventricular hypertrophy was measured, as defined by the Fulton index (weight of RV/(LV+S)) (n=9-14 rats per group). d) The neointima to media ratio of pulmonary arteries (<90 $\mu$ m in diameter) from lung sections of 
SuHx-treated rats was determined by morphometric analysis of pulmonary vessels following Elastica van Giesson staining ( $\mathrm{n}=9-14$ rats per group). Scale bar: $20 \mu \mathrm{m}$. e) Immunofluorescent staining for Ki-67 (green) was performed in small pulmonary arteries from vehicle- and 6-MP-treated rats. SMA (red) and nuclei (DAPI; blue) were co-stained with Ki-67 (images representative of $n=9-14$ lungs per group). f) Mean cardiomyocyte CSA was assessed in the RV from vehicle- and 6-MP-treated rats. Scale bar: $20 \mu \mathrm{m}$. Quantification of CSA is shown in the bar graph next to the images ( $n=9-14$ rats per group). g) Picrosirius red staining was performed to assess the fibrosis in the RV from vehicle- and 6-MP-treated rats. Scale bar: $80 \mu \mathrm{m}$. Quantification of fibrosis is shown in the bar graph next to the images (n=9-14 rats per group). *: $p<0.05$. The t-test was used for comparisons between two groups. Multiple comparisons were assessed by one-way ANOVA followed by Bonferroni's post hoc test. Data are presented as mean \pm SEM.

Expression of Nur77 has been implicated in several vascular diseases, including atherosclerosis, cardiac hypertrophy and coronary restenosis [20-28], where Nur77 inhibits proliferation of endothelial cells, smooth muscle cells, pericytes and fibroblasts, while at the same time suppressing inflammation [23, 29, 35, 45]. Proliferation and inflammation are key features of pulmonary vascular remodelling in PAH [13-17]. Several known triggers of PAH (e.g. DNA damage, inflammation, hypoxia, TGF- $\beta$ signalling, histone acetylation and disturbed flow) have been shown to regulate Nur77 expression and activity in a cell- and context-dependent manner [30, 46-48]. Indeed, in the present study, we found that inhibition of histone deacetylases increased Nur77 expression in PAH MVECs (supplementary figure S1f). In addition, post-translational modifications such as phosphorylation and ubiquitination were also shown to regulate Nur77 expression/activity. Indeed, a number of kinases have been shown to phosphorylate and/or interact with Nur77 directly to modulate its activity [22]. Nur77 agonist 6-MP increases the activity of Nur77 and its family members Nurr1 and Nor1 via direct modulation of the activation function (AF)-1 domain which is located within the N-terminal regulatory (A/B) domain of Nur77 [32]. Interestingly, this increase in activity of Nur77 by 6-MP does not require the ligand-binding domain of Nur77. It has also been demonstrated that 6-MP can modulate the activity of the Nur77 coactivator TRAP220, which directly interacts with the AF-1 domain of Nur77 in vivo [49]. Therefore, 6-MP can regulate the expression and activity of Nur77 via direct modulation of the AF-1 domain and/or activation of its coactivator TRAP220 in a celland context-dependent manner. However, as it is beyond the scope of this study, this has not been investigated here.

A key mechanism by which Nur77 and its agonist 6-MP inhibit cell proliferation is through attenuation of cyclin D1, a known target gene of Nur77 [25, 33, 50]. Indeed, we demonstrated that restoration of Nur77 in PAH MVECs reduced cyclin D1 expression and cellular proliferation. While the role of Nur77 was not previously studied in relevant cell populations from patients with $\mathrm{PAH}$, two recent studies demonstrated that Nur77 exhibits anti-proliferative effects in rat PASMCs via the STAT3-Pim-1-NFAT pathway and human PASMCs via the axin $2-\beta$-catenin signalling pathway $[28,29]$. Consistent with this, in the present study, we also found that 6-MP directly decreases proliferation of healthy human PASMCs (supplementary figure S1e). The communication between endothelial cells and smooth muscle cells plays an essential role in the pathogenesis of PAH. In our study, we found that Nur77 is a key player at the interplay between these cells since the secretome of the diseased PAECs stimulates PASMC growth. In addition to effects on endothelial cell and smooth muscle cell proliferation, Nur77 and its activator 6-MP are known to inhibit the NF- $\kappa B$ pathway, thereby reducing the production of several cytokines $[21,24,27]$. Here, we confirm that 6-MP decreases the secretion of inflammatory cytokines in pulmonary MVECs, and also in serum and lung tissue of 6-MP-treated SuHx rats.

While the effects of Nur77 on vascular proliferation and inflammation were possibly exerted through interaction with multiple pathways, we provide the first evidence that at least some of this effect in PAH is through control of the BMPR2 gene. Germline mutation in BMPR2 is the strongest known genetic risk factor associated with $\mathrm{PAH} ; 40 \%$ of all subjects carrying mutations resulting in haploinsufficiency will ultimately develop HPAH $[4,17,44,51,52]$. Both BMPR2 and BMP signalling are reduced even in IPAH patients $[4,6]$. Loss of BMPR2 has been linked to increased inflammation and proliferation of pulmonary endothelial cells, and it has been suggested that restoring BMP signalling might be beneficial for the treatment of PAH patients $[18,19]$. Indeed, enhanced BMP signalling by BMP9 treatment reduced the development of PAH in animal models [37]. Previously, Nur77 was shown to modulate TGF- $\beta$ signalling in a cell- and context-dependent manner [30,31]. Our data provide the first in vitro and in vivo evidence that Nur77 controls BMP signalling as well. Our findings imply that Nur77, via the BMPR2-SMAD-Id1 axis, may significantly promote endothelial cell function in HPAH and IPAH. Therefore, activation of Nur77 can serve as a novel therapeutic approach for HPAH and other PAH patients with impaired BMP signalling.

Whether using a prevention or reversal strategy, oral administration of 6-MP markedly reduced RVSP and improved RV function in the SuHx PH rat model. The haemodynamic effects of 6-MP were associated with markedly reduced proliferation and inflammation, and reversed abnormal vascular remodelling. Crucially, the beneficial effect of 6-MP was associated with normalisation of Nur77 expression and BMP 

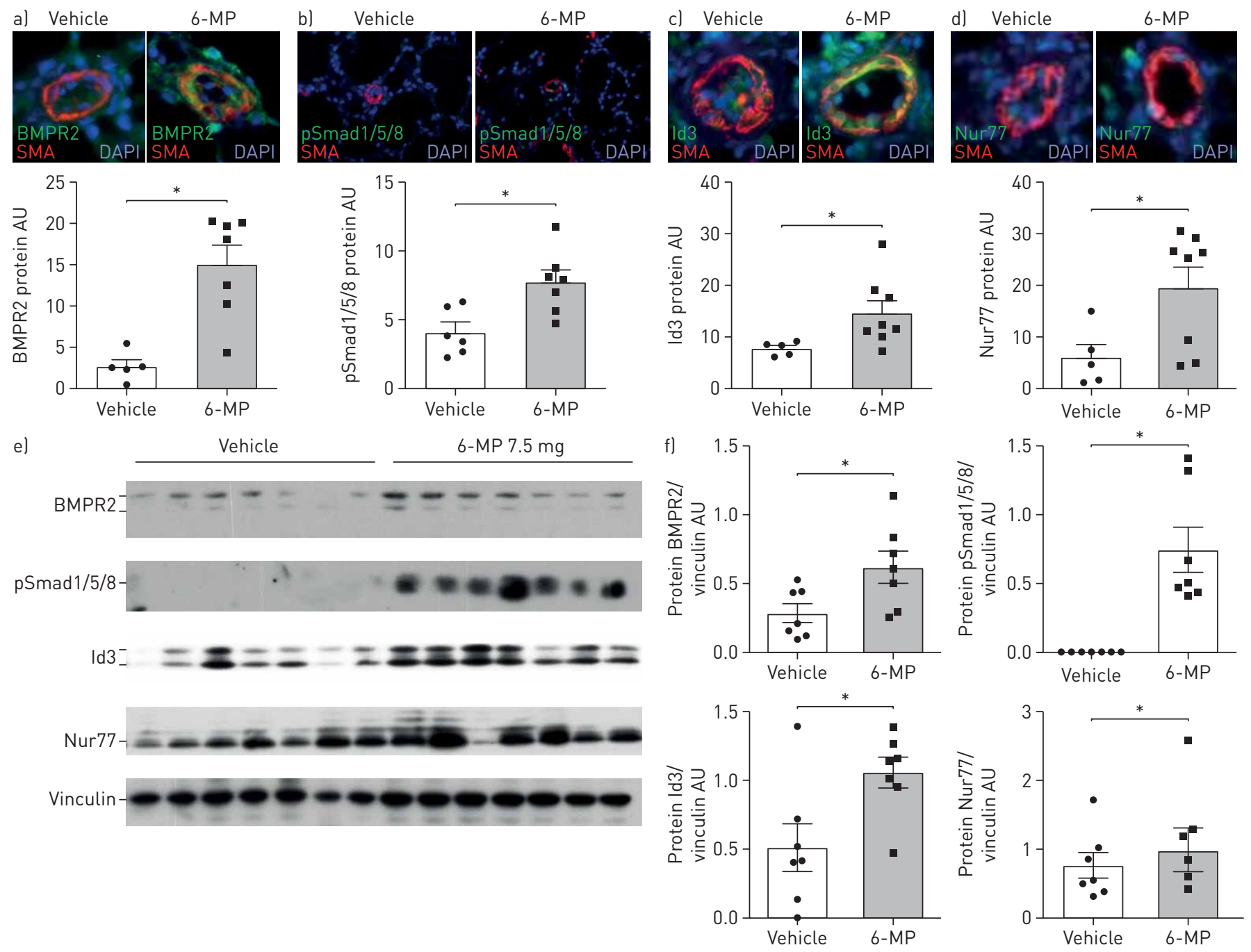

g)

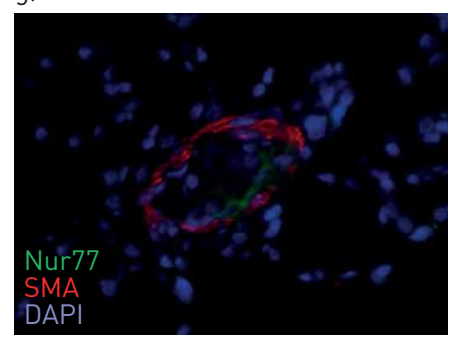

6-MP $7.5 \mathrm{mg}$

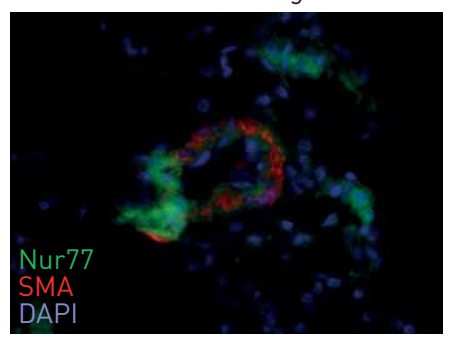

h)

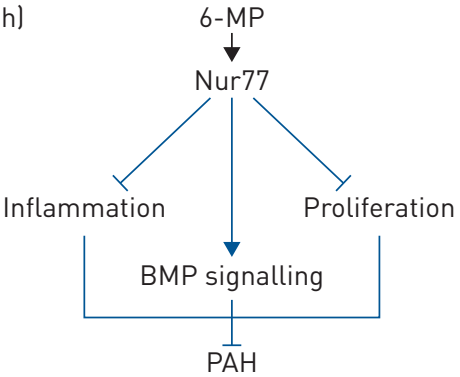

FIGURE 6 6-Mercaptopurine (6-MP) enhances Nur77 expression and amplifies bone morphogenetic protein (BMP) signalling in vivo. BMPR2: BMP receptor 2; SMA: $\alpha$-smooth muscle actin; DAPI: 4',6-diamidino-2-phenylindole; pSmad1/5/8: phosphorylated Smad1/5/8; AU: arbitrary units; PAH: pulmonary arterial hypertension. a-d) Immunofluorescent staining for a) BMPR2 (green), b) pSmad1/5/8 (green), c) Id3 (green) and d) Nur77 (green) was performed in small pulmonary arteries from vehicle- and 6-MP-treated rats from the prevention study. SMA (red) defines smooth muscle cells and DAPI (blue) was used to counterstain nuclei ( $\mathrm{n}=6-8$ lungs per group). e) Western blots showing BMPR2, pSmad1/5/8, Id3 and Nur77 with vinculin as a loading control in total lung lysates from the Sugen/hypoxia rats treated with vehicle and $6-\mathrm{MP}\left(7.5 \mathrm{mg} \cdot \mathrm{kg}^{-1} \cdot \mathrm{day}^{-1}\right.$ ) ( $\mathrm{n}=7$ lungs per group). f) Relative densitometric analyses of the Western blots showing BMPR2, pSmad1/5/8, Id 3 and Nur77 (n=7 lungs per group). g) Immunofluorescent staining for Nur77 (green) was performed in small pulmonary arteries from vehicle- and $6-\mathrm{MP}\left(7.5 \mathrm{mg} \cdot \mathrm{kg}^{-1} \cdot \mathrm{day}^{-1}\right)$-treated rats. SMA (red) and nuclei (DAPI; blue) were co-stained with Nur77 ( $=8$ lungs per group). *: $p<0.05$. The t-test was used for comparisons between two groups. Multiple comparisons were assessed by one-way ANOVA followed by Bonferroni's post hoc test. Data are presented as mean \pm SEM. h) Proposed mechanism of Nur77 6-MP in the reversal of PAH. 6-MP increases the transcriptional activity of Nur77 which 1) inhibits inflammation through blocking the NF-kB pathway, 2) blocks proliferation through inhibition of cell cycle proteins and 3) enhances BMP signalling via activation of the BMPR2-pSmad1/5/8-Id1/3 axis by enhancing BMPR2 expression. Abnormal proliferation and excessive inflammation with impaired BMP signalling leads to initiation and progression of PAH. Activation of Nur77 via clinically approved small-molecule activators such as the Nur77 agonist 6-MP reverses these aberrant changes and attenuates $\mathrm{PAH}$. 
signalling. Taking into account the observed beneficial effect of 6-MP in SuHx rats in this short treatment period, prolonged treatment with 6-MP may even result in a stronger reversal of PAH. Importantly, we observed no immunosuppression at the dose given in the 6-MP-treated animals in both the prevention and reversal study. Although no side-effects were observed in these pre-clinical models and 6-MP is already used in several clinical conditions in patients [21,35,44], future research should focus on testing 6-MP in combination with other PAH drugs as well as the development of lung-specific delivery methods [53] to achieve efficient pulmonary efficacy at low drug concentrations.

Despite recent advances in PAH research, there is still no cure for this deadly disease. Endothelial cell dysfunction is a crucial element in the initiation and pathogenesis of $\mathrm{PAH}$, and our data provide evidence that Nur77 is a novel positive regulator of pulmonary MVEC function and thereby inhibits adverse vascular remodelling in PAH. Specifically, Nur77 exhibits significant anti-proliferative and anti-inflammatory effects and augments BMP signalling in vitro and in vivo. In conclusion, 6-MP displays beneficial effects in vitro and in vivo, and this well-known drug provides an innovative treatment opportunity for patients suffering from the so far untreatable disease PAH.

Acknowledgements: We thank Martijn Rabelink (Leiden University Medical Center, Leiden, The Netherlands) for providing short hairpin RNAs targeting Nur77.

Support statement: We acknowledge support from the Netherlands CardioVascular Research Initiative: the Dutch Heart Foundation, Dutch Federation of University Medical Centers, the Netherlands Organization for Health Research and Development, and the Royal Netherlands Academy of Sciences grant 2012-08 awarded to the Phaedra consortium (www.phaedraresearch.nl). We also acknowledge support for K. Kurakula by the Grants4Targets (Bayer AG) grant 2016-03-1554 and by the Dutch Lung Foundation (Longfonds) grant 5.2.17.198J0. Funding information for this article has been deposited with the Crossref Funder Registry.

Conflict of interest: None declared.

\section{References}

1 Tuder RM, Archer SL, Dorfmuller P, et al. Relevant issues in the pathology and pathobiology of pulmonary hypertension. J Am Coll Cardiol 2013; 62: 25 Suppl., D4-12.

2 D'Alonzo GE, Barst RJ, Ayres SM, et al. Survival in patients with primary pulmonary hypertension. Results from a national prospective registry. Ann Intern Med 1991; 115: 343-349.

3 Galiè N, Humbert M, Vachiery JL, et al. 2015 ESC/ERS Guidelines for the diagnosis and treatment of pulmonary hypertension: The Joint Task Force for the Diagnosis and Treatment of Pulmonary Hypertension of the European Society of Cardiology (ESC) and the European Respiratory Society (ERS): Endorsed by: Association for European Paediatric and Congenital Cardiology (AEPC), International Society for Heart and Lung Transplantation (ISHLT). Eur Respir J 2015; 46: 903-975.

4 Morrell NW, Adnot S, Archer SL, et al. Cellular and molecular basis of pulmonary arterial hypertension. $J$ Am Coll Cardiol 2009; 54: 1 Suppl., S20-S31.

5 Yang J, Davies RJ, Southwood M, et al. Mutations in bone morphogenetic protein type II receptor cause dysregulation of Id gene expression in pulmonary artery smooth muscle cells: implications for familial pulmonary arterial hypertension. Circ Res 2008; 102: 1212-1221.

6 Atkinson C, Stewart S, Upton PD, et al. Primary pulmonary hypertension is associated with reduced pulmonary vascular expression of type II bone morphogenetic protein receptor. Circulation 2002; 105: 1672-1678.

7 Voelkel NF, Gomez-Arroyo J, Abbate A, et al. Pathobiology of pulmonary arterial hypertension and right ventricular failure. Eur Respir J 2012; 40: 1555-1565.

8 Perros F, Bonnet S. Bone morphogenetic protein receptor type II and inflammation are bringing old concepts into the new pulmonary arterial hypertension world. Am J Respir Crit Care Med 2015; 192: 777-779.

9 Alastalo TP, Li M, de Jesus Perez V, et al. Disruption of PPARgamma/beta-catenin-mediated regulation of apelin impairs BMP-induced mouse and human pulmonary arterial EC survival. J Clin Invest 2011; 121: 3735-3746.

10 Hong KH, Lee YJ, Lee E, et al. Genetic ablation of the BMPR2 gene in pulmonary endothelium is sufficient to predispose to pulmonary arterial hypertension. Circulation 2008; 118: 722-730.

11 Yuan K, Shao NY, Hennigs JK, et al. Increased pyruvate dehydrogenase kinase 4 expression in lung pericytes is associated with reduced endothelial-pericyte interactions and small vessel loss in pulmonary arterial hypertension. Am J Pathol 2016; 186: 2500-2514.

12 Zhang $\mathrm{H}$, Wang $\mathrm{D}$, Li $\mathrm{M}$, et al. The metabolic and proliferative state of vascular adventitial fibroblasts in pulmonary hypertension is regulated through a MiR-124/PTBP1/PKM axis. Circulation 2017; 136: 2468-2485.

13 Le Hiress M, Tu L, Ricard N, et al. Proinflammatory signature of the dysfunctional endothelium in pulmonary hypertension. Role of the macrophage migration inhibitory factor/CD74 complex. Am J Respir Crit Care Med 2015; 192: 983-997.

14 Meloche J, Renard S, Provencher S, et al. Anti-inflammatory and immunosuppressive agents in PAH. Handb Exp Pharmacol 2013; 218: 437-476.

15 Savai R, Pullamsetti SS, Kolbe J, et al. Immune and inflammatory cell involvement in the pathology of idiopathic pulmonary arterial hypertension. Am J Respir Crit Care Med 2012; 186: 897-908.

16 Toshner M, Voswinckel R, Southwood M, et al. Evidence of dysfunction of endothelial progenitors in pulmonary arterial hypertension. Am J Respir Crit Care Med 2009; 180: 780-787.

17 Voelkel NF, Tamosiuniene R, Nicolls MR. Challenges and opportunities in treating inflammation associated with pulmonary hypertension. Expert Rev Cardiovasc Ther 2016; 14: 939-951.

18 Majka S, Hagen M, Blackwell T, et al. Physiologic and molecular consequences of endothelial Bmpr2 mutation. Respir Res 2011; 12: 84. 
19 Xiong J. BMPR2 spruces up the endothelium in pulmonary hypertension. Protein Cell 2015; 6: 703-708.

20 Kurakula K, Hamers AA, de Waard V, et al. Nuclear receptors in atherosclerosis: a superfamily with many "Goodfellas". Mol Cell Endocrinol 2013; 368: 71-84.

21 Kurakula K, Hamers AA, van Loenen $\mathrm{P}$, et al. 6-Mercaptopurine reduces cytokine and Muc5ac expression involving inhibition of NFkappaB activation in airway epithelial cells. Respir Res 2015; 16: 73.

22 Kurakula K, Koenis DS, van Tiel CM, et al. NR4A nuclear receptors are orphans but not lonesome. Biochim Biophys Acta 2014; 1843: 2543-2555.

23 Kurakula K, van der Wal E, Geerts D, et al. FHL2 protein is a novel co-repressor of nuclear receptor Nur77. J Biol Chem 2011; 286: 44336-44343.

24 Kurakula K, Vos M, Logiantara A, et al. Nuclear receptor Nur77 attenuates airway inflammation in mice by suppressing NF-kappaB activity in lung epithelial cells. J Immunol 2015; 195: 1388-1398.

25 Liu Y, Zhang J, Yi B, et al. Nur77 suppresses pulmonary artery smooth muscle cell proliferation through inhibition of the STAT3/Pim-1/NFAT pathway. Am J Respir Cell Mol Biol 2014; 50: 379-388.

26 Medzikovic L, Schumacher CA, Verkerk AO, et al. Orphan nuclear receptor Nur77 affects cardiomyocyte calcium homeostasis and adverse cardiac remodelling. Sci Rep 2015; 5: 15404.

27 Qin Q, Chen M, Yi B, et al. Orphan nuclear receptor Nur77 is a novel negative regulator of endothelin-1 expression in vascular endothelial cells. J Mol Cell Cardiol 2014; 77: 20-28.

28 You B, Jiang YY, Chen S, et al. The orphan nuclear receptor Nur77 suppresses endothelial cell activation through induction of IkappaBalpha expression. Circ Res 2009; 104: 742-749.

29 Nie X, Tan J, Dai Y, et al. Nur77 downregulation triggers pulmonary artery smooth muscle cell proliferation and migration in mice with hypoxic pulmonary hypertension via the Axin2-beta-catenin signaling pathway. Vascul Pharmacol 2016; 87: 230-241.

30 Palumbo-Zerr K, Zerr P, Distler A, et al. Orphan nuclear receptor NR4A1 regulates transforming growth factor-beta signaling and fibrosis. Nat Med 2015; 21: 150-158.

31 Zhou F, Drabsch Y, Dekker TJ, et al. Nuclear receptor NR4A1 promotes breast cancer invasion and metastasis by activating TGF-beta signalling. Nat Commun 2014; 5: 3388.

32 Wansa KD, Harris JM, Yan G, et al. The AF-1 domain of the orphan nuclear receptor NOR-1 mediates trans-activation, coactivator recruitment, and activation by the purine anti-metabolite 6-mercaptopurine. $J$ Biol Chem 2003; 278: 24776-24790.

33 Marinkovic G, Kroon J, Hoogenboezem M, et al. Inhibition of GTPase Rac1 in endothelium by 6-mercaptopurine results in immunosuppression in nonimmune cells: new target for an old drug. J Immunol 2014; 192: 4370-4378

34 Weigel G, Griesmacher A, DeAbreu RA, et al. Azathioprine and 6-mercaptopurine alter the nucleotide balance in endothelial cells. Thromb Res 1999; 94: 87-94.

35 Pires NM, Pols TW, de Vries MR, et al. Activation of nuclear receptor Nur77 by 6-mercaptopurine protects against neointima formation. Circulation 2007; 115: 493-500.

36 Szulcek R, Happe CM, Rol N, et al. Delayed microvascular shear adaptation in pulmonary arterial hypertension. role of platelet endothelial cell adhesion molecule-1 cleavage. Am J Respir Crit Care Med 2016; 193: 1410-1420

37 Long L, Ormiston ML, Yang X, et al. Selective enhancement of endothelial BMPR-II with BMP9 reverses pulmonary arterial hypertension. Nat Med 2015; 21: 777-785.

38 da Silva Goncalves Bos D, Van Der Bruggen CE, Kurakula K, et al. Contribution of impaired parasympathetic activity to right ventricular dysfunction and pulmonary vascular remodeling in pulmonary arterial hypertension. Circulation 2017; 137: 910-924.

39 de Raaf MA, Kroeze Y, Middelman A, et al. Serotonin transporter is not required for the development of severe pulmonary hypertension in the Sugen hypoxia rat model. Am J Physiol Lung Cell Mol Physiol 2015; 309: L1164-L1173.

40 Provencher S, Archer SL, Ramirez FD, et al. Standards and methodological rigor in pulmonary arterial hypertension preclinical and translational research. Circ Res 2018; 122: 1021-1032.

41 Korchynskyi O, ten Dijke P. Identification and functional characterization of distinct critically important bone morphogenetic protein-specific response elements in the Id1 promoter. J Biol Chem 2002; 277: 4883-4891.

42 Zhan $\mathrm{Y}, \mathrm{Du} \mathrm{X}$, Chen $\mathrm{H}$, et al. Cytosporone B is an agonist for nuclear orphan receptor Nur77. Nat Chem Bio 2008; 4: 548-556.

43 Taraseviciene-Stewart L, Kasahara Y, Alger L, et al. Inhibition of the VEGF receptor 2 combined with chronic hypoxia causes cell death-dependent pulmonary endothelial cell proliferation and severe pulmonary hypertension. FASEB J 2001; 15: 427-438.

44 Domenech E, Nos P, Papo M, et al. 6-mercaptopurine in patients with inflammatory bowel disease and previous digestive intolerance of azathioprine. Scand J Gastroenterol 2005; 40: 52-55.

45 Huo Y, Yi B, Chen M, et al. Induction of Nur77 by hyperoside inhibits vascular smooth muscle cell proliferation and neointimal formation. Biochem Pharmacol 2014; 92: 590-598.

46 To SK, Zeng WJ, Zeng JZ, et al. Hypoxia triggers a Nur77- $\beta$-catenin feed-forward loop to promote the invasive growth of colon cancer cells. Br J Cancer 2014; 110: 935-945.

47 Xie X, Song X, Yuan S, et al. Histone acetylation regulates orphan nuclear receptor NR4A1 expression in hypercholesterolaemia. Clin Sci 2015; 129: 1151-1161.

48 Dequiedt F, Kasler H, Fischle W, et al. HDAC7, a thymus-specific class II histone deacetylase, regulates Nur77 transcription and TCR-mediated apoptosis. Immunity 2003; 18: 687-698.

49 Wansa KD, Muscat GE. TRAP220 is modulated by the antineoplastic agent 6-mercaptopurine, and mediates the activation of the NR4A subgroup of nuclear receptors. J Mol Endocrinol 2005; 34: 835-848.

50 Nomiyama T, Zhao Y, Gizard F, et al. Deficiency of the NR4A neuron-derived orphan receptor-1 attenuates neointima formation after vascular injury. Circulation 2009; 119: 577-586.

51 Deng Z, Morse JH, Slager SL, et al. Familial primary pulmonary hypertension (gene PPH1) is caused by mutations in the bone morphogenetic protein receptor-II gene. Am J Hum Genet 2000; 67: 737-744.

52 van der Bruggen CE, Happe CM, Dorfmuller P, et al. Bone morphogenetic protein receptor type 2 mutation in pulmonary arterial hypertension: a view on the right ventricle. Circulation 2016; 133: 1747-1760.

53 Yin $\mathrm{Y}, \mathrm{Wu} \mathrm{X}$, Yang Z, et al. The potential efficacy of R8-modified paclitaxel-loaded liposomes on pulmonary arterial hypertension. Pharm Res 2013; 30: 2050-2062. 